This document is the Accepted Manuscript version of a Published Work that appeared in final form in The Journal of Molecular Spectroscopy, copyright (C) Elsevier Inc., under the citation mentioned below:

Analysis of high resolution FTIR spectra from synchrotron sources using evolutionary algorithms ( $\odot 2015$ by Jennifer van Wijngaarden, Durell Desmond, W. Leo Meerts is licensed under CC BY-NC-ND 4.0

DOI: https://doi.org/10.1016/j.jms.2015.04.002

\title{
Analysis of high resolution FTIR spectra from synchrotron sources using Evolutionary Algorithms
}

Jennifer van Wijngaarden ${ }^{\mathrm{a} *}$, Durell Desmond ${ }^{\mathrm{a}}$ and W. Leo Meerts ${ }^{\mathrm{b}}$

${ }^{a}$ Department of Chemistry, University of Manitoba, Winnipeg Manitoba, R3T 2N2

Canada

${ }^{\mathrm{b}}$ Radboud University, Molecular and Biophysics Group, Institute for Molecules and Materials, P.O. Box 9010, 6500 GL Nijmegen, The Netherlands

*Corresponding author

Email: vanwijng@cc.umanitoba.ca

Phone: (204)474-8379

Fax: (204)474-7608 


\begin{abstract}
Room temperature Fourier transform infrared spectra of the four-membered heterocycle trimethylene sulfide were collected with a resolution of $0.00096 \mathrm{~cm}^{-1}$ using synchrotron radiation from the Canadian Light Source from 500 to $560 \mathrm{~cm}^{-1}$. The inplane ring deformation mode $\left(v_{13}\right)$ at $\sim 529 \mathrm{~cm}^{-1}$ exhibits dense rotational structure due to the presence of ring inversion tunneling and leads to a doubling of all transitions. Preliminary analysis of the experimental spectrum was pursued via traditional methods involving assignment of quantum numbers to individual transitions in order to conduct least squares fitting to determine the spectroscopic parameters. Following this approach, the assignment of 2358 transitions led to the experimental determination of an effective Hamiltonian. This model describes transitions in the $\mathrm{P}$ and $\mathrm{R}$ branches to $\mathrm{J}^{\prime}=60$ and $\mathrm{K}_{\mathrm{a}}{ }^{\prime}=10$ that connect the tunneling split ground and vibrationally excited states of the $v_{13}$ band although a small number of low intensity features remained unassigned. The use of evolutionary algorithms (EA) for automated assignment was explored in tandem and yielded a set of spectroscopic constants that re-create this complex experimental spectrum to a similar degree. The EA routine was also applied to the previously wellunderstood ring puckering vibration of another four-membered ring, azetidine [ Molecular Spectroscopy, 264, 105-110]. This test provided further evidence of the robust nature of the EA method when applied to spectra for which the underlying physics is well understood.
\end{abstract}

KEYWORDS: rovibrational spectroscopy; evolutionary algorithms; genetic algorithms; synchrotron; infrared; azetidine; trimethylene sulfide 


\section{Introduction}

In recent years, the use of synchrotron light as a radiation source for high resolution gas phase infrared spectroscopy has grown in popularity but the pool of expert users of such beamlines remains small compared to those for other synchrotron-based techniques. One challenge is the time-consuming nature of the data analysis that is required to account for the thousands of rovibrational transitions that typically fall within each band. Methods that automate the assignment of dense, rovibrational spectra hold promise to reduce this analysis bottleneck and would allow spectroscopists to focus their attention instead on a broader range of interesting, scientific questions. In this article, we test the application of evolutionary algorithms (EA) for analyzing both simple and complex far infrared spectra obtained at the Canadian Light Source (CLS) synchrotron.

EAs are heuristics that mimic natural selection processes in order to automatically find the global solution to an optimization problem. In the context of spectroscopy, EAs are designed to generate a set of spectroscopic parameters that best simulate an experimental spectrum without assigning quantum numbers to individual transitions at the outset. To do this, the individual molecular parameters or genes that comprise each chromosome (or set of molecular parameters) are adjusted in each iteration of the analysis using concepts derived from genetics such as inheritance, mutation and crossover. The performance of the EA is assessed by correlating frequency and intensity information between the simulated and experimental spectrum through the use of a fitness function. ${ }^{1}$ A typical fitness function is the inner product between the two vectors representing the experimental and calculated spectrum. This inner product has it maximum if the two spectra are identical. Various EA strategies have been developed and 
tested for their robustness in dealing with high resolution spectra including Laser Induced Fluorescence, ${ }^{2,3}$ FTIR spectroscopy, ${ }^{4}$ Room temperature UV absorption spectroscopy ${ }^{5}$ and NMR spectroscopy. ${ }^{6,7}$ While the application of EAs to analyze rotationally-resolved spectra has been successfully demonstrated for a range of spectroscopic techniques, it has not yet been tested for high resolution room temperature FTIR spectra. The high resolution FTIR spectrometers that are currently available at national synchrotron facilities provide spectra with resolution better than $0.001 \mathrm{~cm}^{-1}$ and thus allow a large number of individual transitions to be discerned rather than profiles resulting from groups of closely-space transitions. As a result, even very minor perturbations of energy levels are easily detected. Synchrotron-based measurements also allow routine access to wavelengths well into the far infrared region where vibrational frequencies are less accurately predicted and the observed spectra of even small molecules are very congested by hotbands or other complications. To test the robustness of the EA strategy for analyzing far infrared spectra, we have applied this technique to i) the ring puckering band of azetidine near $207 \mathrm{~cm}^{-1}$ which was previously analyzed ${ }^{8}$ and known to be wellbehaved and ii) the newly recorded in-plane ring deformation band of trimethylene sulfide (TMS) near $529 \mathrm{~cm}^{-1}$ which is investigated here for the first time.

Trimethylene sulfide (TMS) or thietane is a four-membered heterocycle with a non-planar heavy atom skeleton as shown in Figure 1. TMS has been investigated in the microwave, ${ }^{9,10,11}$ millimeterwave ${ }^{12}$ and infrared regions ${ }^{13,14,15,16}$ and these studies have largely focused on the ground and ring puckering vibrational states. Although this and related four-membered rings such as oxetane have been under spectroscopic scrutiny for half a century, modern high resolution FTIR instruments have enabled their study in 
greater detail. ${ }^{17,18}$ Synchrotron-based FTIR spectra have been used, for example, to derive the ring puckering potential of 3 -oxetanone $\left(\mathrm{c}-\mathrm{C}_{2} \mathrm{H}_{4}(\mathrm{CO}) \mathrm{O}\right)^{19}$ using its hotbands at $\sim 140 \mathrm{~cm}^{-1}$, to characterize the Coriolis interaction between overlapping bands in 2oxetanone $^{20}$ and to describe the coupling of $\mathrm{NH}$ inversion tunneling with ring inversion in the low-lying vibrations of azetidine $\left(\mathrm{c}-\mathrm{C}_{3} \mathrm{~N}_{6} \mathrm{NH}\right){ }^{8}$

In the case of TMS, the pure rotational spectrum itself was complicated by the presence of ring inversion tunneling through a planar configuration corresponding to a barrier of $\sim 274.2 \mathrm{~cm}^{-1}$ above the equilibrium, puckered geometry. ${ }^{10}$ The $a$-type rotational transitions were thus tunneling doubled and assigned to the two ring inversion components of the ground vibrational state $\left(0^{+}\right.$and $\left.0^{-}\right)$. A few transitions connecting the ground state inversion components were also observed and followed $c$-type selection rules. Their observation allowed the determination of the energy gap between inversion components $0^{+}$and $0^{-}$which was determined to be $8232 \mathrm{MHz}\left(\sim 0.27 \mathrm{~cm}^{-1}\right)$. The rotational spectrum was perturbed by the mixing of pairs of energy levels in the two inversion manifolds as their separation was within the range of rotational energy spacing of TMS. In order to analyze the spectrum, Coriolis-like interaction terms were incorporated into the Hamiltonian to describe the mixing of the levels in the $0^{+}$and $0^{-}$ states. ${ }^{10,12}$ No rotationally-resolved vibrational spectra of TMS have been reported before now.

\section{Evolutionary algorithms}

In the past decades, computational tools have been developed that are able to handle complex multi-parameter optimizations intelligently and within an acceptable investment in computer time. The use of evolutionary algorithms (EAs) for solving such 
highly nonlinear and complex processes in science and engineering has become widespread. Although they are conceptually simple, their ability to avoid local optima and instead find the global optimum is remarkable and makes them suitable to handle complex optimization problems. EAs represent a set of general-purpose probabilistic search methods based on natural evolution. These algorithms mimic the concepts of natural reproduction and selection processes. The basic idea in EAs is to create an artificial environment that encodes the search problem into biology-like terms. From a spectroscopic point of view, the EA approach reproduces this behavior to fit an experimental spectrum with a model based upon transitions between spin energy levels calculated as eigenvalues of the Hamiltonian. Each of the molecular parameters can be thought of as a gene, encoded in a binary or real type. The vector of all genes, which contains all molecular parameters, is called a chromosome. The rovibrational spectra were fit to an asymmetric rotor Hamiltonian by using the covariance matrix adaptation evolutionary strategy (CMA-ES) ${ }^{21},{ }^{22}$ which was found to be an invaluable tool for the analysis of many different types of spectra. While classical genetic algorithms aim to find a solution in the parameter space by randomly combining information from a set of trial solutions, the CMA-ES algorithm can sense in which direction the quality of a fit increases. In the first step, the CMA-ES algorithm generates trial solutions (offspring) by using a random distribution around some starting point (parent) each consisting of the complete parameter set which is necessary to simulate the spectrum. Offspring with higher fitness than the parent are kept and used to compute the next generation. The CMA-ES algorithm makes use of the parameter correlation matrix for successive changes in the parents (mutations). This means that if for some parameter a parent has evolved in 
the same direction for several generations, resulting in a positive correlation, the most likely solution is assumed to be further in that direction and the next parameter mutation will be larger. Correspondingly, two anti-correlated mutations will lead to a smaller mutation. Compared to classical genetic algorithms, this procedure has been shown to lead to faster convergence of the parameters. ${ }^{23}$ The CMA-ES method is essentially a brute-force method that relies on recent developments in computer technology which have been geared towards the analysis of complex spectra. The CMA-ES method has been shown to be an extremely useful tool for spectral analysis.

\subsection{Testing the evolutionary algorithm on azetidine}

As a first test of the EA approach, we applied this method to the previously reported spectrum of the ring puckering mode of azetidine $\left(c-\mathrm{C}_{3} \mathrm{H}_{6} \mathrm{NH}\right)$ centered at 207 $\mathrm{cm}^{-1}$ which was recorded at the CLS. ${ }^{8}$ During this vibration, the amine hydrogen moves from axial to equatorial position as the ring backbone flexes which corresponds to a change in dipole moment along the a-axis as the projection of the $\mathrm{NH}$ bond varies in this coordinate. This leads to a-type rovibrational selection rules and the observed spectrum was not complicated by ring inversion tunneling as the barrier between equivalent forms (requiring both ring inversion and NH inversion) is quite high $\left(\sim 2200 \mathrm{~cm}^{-1}\right)$. The analysis of the 3278 assigned transitions has been described and the room temperature spectrum actually contains transitions from a very large number of rotational states up to $\mathrm{J}$ values of 70. Almost all transitions are unblended and therefore this spectrum is suited excellently for a test of the EA. As mentioned, the frequencies and intensities of the experimental and the calculated spectrum are matched in a global manner in the EA. The calculated spectrum is obtained from a model Hamiltonian, but in the fitting process no 
quantum numbers have to be assigned. This automatic search together with the global search of the parameter space, form the strength of the EA method. After the EA fit has converged, the results include both the values of the molecular parameters from the Hamiltonian as well as the full assignment of all individual transitions. In order for the method to reach convergence successfully within acceptable CPU-time, reasonable ranges for the molecular parameters must be defined beforehand.

For the azetidine molecule, we found that with the CMA-ES strategy, we could extend the search ranges around the estimated values for the rotational constants to \pm $0.002 \mathrm{~cm}^{-1}$, for the band center $\left(v_{0}\right)$ to $\pm 0.02 \mathrm{~cm}^{-1}$ and for the distortion constants to \pm 0.6 $\mathrm{x} 10^{-6} \mathrm{~cm}^{-1}$. For the latter group of parameters this meant that no estimates were needed for the distortion constants, since the values fall well within the search regions. Despite the large search ranges for the parameters, the fit converged rapidly. The CMA-ES procedure included 250 individuals and the number of fitted parameters was 17 . The convergence set in after 50 generations and was completed after 150 generations. The wall clock time needed was five minutes with the program running on a cluster of only 30 nodes.

Figure 2 compares a portion of the R-branch of the experimental spectrum with that generated from the EA-derived parameters showing the excellent agreement. This kind of agreement was found over the entire range of the spectrum. The spectroscopic constants obtained from the EA are compared with those determined from the original rovibrational analysis (reference 8) in Table 1 . As the analysis of the $v_{16}$ band was first reported as part of a global fit of three rovibrational bands, it was first necessary to extract the transitions due to this band alone to run an isolated band fit for more direct 
comparison with the EA results obtained here. The errors from the EA analysis as presented in Table 1 were estimated from the covariance matrix with the assumption that the line positions in the spectra are measurable to an accuracy of about half the full linewidth at half maximum. In such an assigned fit, transitions are included that would be used in a typical manual assignment of the spectra; that is transitions with an intensity larger than $5 \%$ of the maximum intensity. The selection of transitions used for the assigned fit can of course be made automatically based on this condition. The number of transitions for azetidine was 3290 with rotational quantum numbers up to $\mathrm{J}=50$. For such an assigned fit the statistical errors in the parameters are well defined (see Appendix $\mathrm{B}$ of reference 2). The agreement shown between the results for the two methods in Table 1 is excellent and well within the uncertainties for the determined parameters. Thus, the speed and accuracy of the EA approach for a single band analysis of a high resolution rovibrational spectrum have been successfully demonstrated for the first time.

\section{Experimental details}

The FTIR spectrum of TMS below $1000 \mathrm{~cm}^{-1}$ was collected on the far infrared beamline of the CLS which is equipped with a Bruker IFS125HR instrument. This spectrometer allows measurement of spectra with a resolution of up to $0.00096 \mathrm{~cm}^{-1}$ when a small aperture is used $\left(1.15 \mathrm{~cm}\right.$ in this case). For the $v_{13}$ band at $\sim 529 \mathrm{~cm}^{-1}$, the spectrometer was outfitted with a $\mathrm{KBr}$ beamsplitter and the helium-cooled $\mathrm{GeCu}$ detector was employed. A sample of TMS was purchased from Santa Cruz Biotechnology (Santa Cruz, CA) and used without further purification. The vapour pressure of the liquid was sufficient to fill the $2 \mathrm{~m}$ multipass cell set to a total absorption path length of $72 \mathrm{~m}$. For 
the $v_{13}$ band, a total of 96 interferograms were recorded at room temperature and averaged over 10 hours using a sample pressure of 185 mTorr.

\section{Spectral analysis and assignment for TMS}

The equilibrium structure of TMS has $\mathrm{C}_{\mathrm{s}}$ symmetry (Figure 1) and in order to account for ring inversion, the molecular symmetry group $\mathrm{C}_{2 \mathrm{v}}$ is used. The symmetry of the various energy levels (rotation, vibration, inversion) follows that described recently for the $v_{10}$ band of silacyclobutane $(\mathrm{SCB}) .{ }^{24}$ As the $v_{13}$ ring deformation of TMS has $\mathrm{A}_{1}$ vibrational symmetry, its infrared spectrum is composed of two sets of a-type rovibrational transitions $\left(0^{+} \rightarrow v_{13}{ }^{+}, 0^{-} \rightarrow v_{13}{ }^{-}\right)$that connect states of the same inversion parity. As seen for SCB, two sets of $c$-type rotation-vibration-inversion transitions $\left(0^{-} \rightarrow\right.$ $\left.1^{+}, 0^{+} \rightarrow 1^{-}\right)$that connect states of opposite inversion parity are also symmetry-allowed.

\subsection{Least squares fitting}

The overall band contour of the $v_{13}$ band of TMS is shown in Figure 3. To identify patterns in the rotational structure, the most intense transitions between 500 and $560 \mathrm{~cm}^{-1}$ were used to construct a Loomis-Wood plot using the Neese software add-in for Igor Pro. ${ }^{25,26} \mathrm{~A}$ sample region is shown in Figure 4 corresponding to the $\mathrm{R}$ branch. The scale chosen highlights progressions sharing common $\mathrm{K}_{\mathrm{a}}$ quantum numbers along various $\mathbf{J}$ values as a vertical series. These correspond to strong $a$-type progressions that connect vibrational levels of the same inversion symmetry $\left(0^{+} \rightarrow v_{13}{ }^{+}\right.$and $\left.0^{-} \rightarrow v_{13^{-}}\right)$. The analogous series in the two tunneling states are identified with the same colour for ease of comparison. The observed pattern was compared with a simulated spectrum based on the previously published ground state parameters for $\mathrm{TMS}^{12}$ to approximate the energy levels. This led to the tentative assignment of the first eight $\mathrm{K}_{\mathrm{a}}$ progressions for each tunneling 
component. Ground state combination differences (GSCDs) were then used to confirm the assignment. The simulated spectrum was then refined and the assignment process continued in an iterative manner until the strongest transitions were included.

A least squares fit of the 2358 infrared transitions was performed using Pickett's SPFIT program and Watson's A-reduced Hamiltonian in the $\mathrm{I}^{\mathrm{r}}$ representation and includes transitions with $\mathrm{J}^{\prime}=8$ to 60 and $\mathrm{K}_{\mathrm{a}}{ }^{\prime}=0$ to $10 .^{27,28}$ The two sets of $a$-type transitions were not sensitive to the energy gap between the inversion split levels $\left(0^{+}\right.$and $\left.0^{-}\right)$nor the parameter describing their interaction $\left(\mathrm{F}_{\mathrm{ac}}, \mathrm{F}_{\mathrm{ac}}{ }^{\prime}\right)$ which were reported in the earlier pure rotational studies. ${ }^{12}$ A similar quality fit was obtained if these parameters were included and fixed to the previously reported values. During the preliminary stages of the analysis, it was also seen that the distortion constants across the four states $\left(0^{+}, 0^{-}, v_{13^{+}}, v_{13^{-}}\right)$were not in close agreement in sign or magnitude and that the A rotational constants were not as well-determined as the others. This suggested that these terms were serving more as effective parameters to reduce the error of the fit. We thus decided to fix A, $\delta_{\mathrm{J}}$ and $\delta_{\mathrm{K}}$ for the $0^{+}$and $0^{-}$states to those values reported in reference 12 . This resulted in a set of spectroscopic parameters that were more consistent across all states. The fit was run both with and without inclusion of the interaction parameters $\left(\mathrm{F}_{\mathrm{ac}}, \mathrm{F}_{\mathrm{ac}}{ }^{\prime}\right)$ from reference 12 and the results are summarized in Table 2.

After the assignment of the strongest $a$-type transitions, a small number of low intensity transitions remained unassigned that were not included in any obvious progressions in the Loomis-Wood plot. These likely correspond to the higher $\mathrm{K}_{\mathrm{a}}$ transitions that are not well-described by the current effective Hamiltonian. Based on 
symmetry, these features could also be $c$-type rotation-vibration-inversion transitions but a convincing assignment could not be found.

\subsection{Evolutionary algorithm approach}

The number of transitions that have to be calculated in the case of TMS is four times as much as for azetidine due to the lower barrier to ring inversion tunneling: $\left(0^{+} \rightarrow\right.$ $\left.v_{13}{ }^{+}\right),\left(0^{-} \rightarrow v_{13}\right),\left(0^{+} \rightarrow v_{13^{-}}\right)$and $\left(0^{-} \rightarrow v_{13}{ }^{+}\right)$. As a result, it was found that the convergence was slower than for azetidine; the onset of the convergence occurred after 175 generations and it was completed after 350 generations. The needed wall clock time was 45 minutes using a cluster of 30 nodes.

After a number of preliminary EA fits to determine the limits of the search ranges, the EA fitting procedure was carried out in two steps. In the first step we fixed the distortion constants for the $0^{-}, 1^{+}$and $1^{-}$states to those of the $0^{+}$state as reported in reference 12. As such, we fixed the A constants for the $0^{+}$and $0^{-}$states to those in reference 12 and restricted the excited vibrational state parameters to be equal $\mathrm{A}\left(\mathrm{v}_{13}{ }^{+}\right)=$ $\mathrm{A}\left(v_{13}{ }^{-}\right)$. In the second step, we released all constraints between the A-rotational constants

and the distortion constants of the + states. Since the distortion constants are just perturbations on the rotational constants and the structure of the molecule in all four states is very similar as follows from the rotational constants, we assumed all corresponding distortion constants to be the same for the + and - states. A total of 20 parameters were thus fit and are summarized in Table 2 along with the results from the SPFIT analyses. The errors from the EA analysis as presented in Table 2 were estimated from the covariance matrix with the assumption that the line positions in the spectra are measurable to an accuracy of $0.25 \times 10^{-3} \mathrm{~cm}^{-1}$, which is about 0.5 of the full line-width at 
half maximum . The errors from the EA analysis as presented in Table 2 were estimated as described above for azetidine with assumption that the line positions in the spectra are measurable to an accuracy of $0.25 \times 10^{-3} \mathrm{~cm}^{-1}$ (half of FWHM). The number of used transitions used for TMS was 6300 with rotational quantum numbers up to $\mathrm{J}=50$.

It was very promising to see that even with the above mentioned approximations for various parameters, the values for the corresponding distortion constants are of the same order of magnitude, as one might expect. For the EA to be successful within an acceptable time span, reasonable estimates for the leading parameters such as rotational constants and rotation-free vibrational frequencies are needed. This is a consequence of the high density of lines in the TMS spectrum which was not an issue with azetidine. Suitable estimates of leading parameters should be obtained from all known experimental information or from $a b$ initio calculations. This highlights the difference between applying the EA method to the two quite different molecular species presented here as little information was needed at the outset of the azetidine analysis.

\section{Discussion and Outlook}

The application of EAs to analyze the $v_{16}$ ring puckering spectrum of azetidine clearly demonstrated the suitability of this approach for FTIR spectra of high resolution $\left(0.00096 \mathrm{~cm}^{-1}\right)$ as demonstrated in Table 1 and Figure 2. This is the most thorough test of this EA method to rovibrational spectra to date as the previous application to FTIR spectra involved transitions that were only partially resolved (line widths $\sim 0.14 \mathrm{~cm}^{-1}$ ). ${ }^{4}$ The current work highlights the potential of EAs for automated spectral assignment of large numbers of individual rovibrational transitions within a matter of minutes and starting from a set of only loosely-determined rotational constants. This study shows that 
a thorough classical assignment as given here is not needed for the EA method to be successful; an assignment of the low $\mathbf{J}$ and $\mathrm{K}$-states would have been enough. For a more complicated molecule like TMS, a more complete classical assignment proved important to compare the various methods.

Based on our experience with TMS, we must warn potential users at the outset that they cannot simply use the EA fit as a "black box" solution. In the analysis of such complicated spectra with a large number of transitions, with intensities that are only slowly varying as a function of the frequency, one has to be extra careful in applying the EA method. Thorough comparison of the shapes of experimental and calculated spectra should be made. The best practice is to perform an EA fit along with a classical analysis in which the latter can be extended step by step using the results of the global EA fit. At the same time, (automatic) assignments found from the EA fit should be compared with those of the classical fit. In this way, it will also be possible to find local perturbations in the spectrum from, for example, Coriolis couplings, an often occurring phenomenon in IR spectra.

Table 2 presents a comparison of the spectroscopic parameters determined via EA analysis of TMS with those obtained from a standard quantum number assignment and least squares analysis. Fits 1 and 2 represent typical rovibrational analyses which either neglect (Fit 1) or include (Fit 2) the interaction parameters for the ground state inversion doublet. Both fits of experimental data successfully account for the observed infrared spectrum up to $\mathrm{J}^{\prime}=60$ and $\mathrm{K}_{\mathrm{a}}{ }^{\prime}=10$ but the assignment could not be extended much further based on these or the EA model. The experimental results are compared with those from the rotational spectrum of Lopez et al. ${ }^{12}$ in Table 3 for the ground state inversion doublet. 
While the parameters derived from the three models are comparable in sign and magnitude across all states, there are notable discrepancies. For example, the $\mathrm{C}$ rotational constant in the $0^{+}$and $0^{-}$states vary across all three methods but follow the same general trend that $\mathrm{C}\left(0^{+}\right)<\mathrm{C}\left(0^{-}\right)$. When the various analyses were attempted without fixing ground state distortion parameters to the values of Lopez et. al., ${ }^{12}$ the discrepancies were greater with distortion constants varying in sign and magnitude across the three methods. This indicates that all models employed in the analysis have provided effective solutions that do not uniquely describe the molecular physics of this band of TMS. Another interesting observation is that the neglect of the interaction parameters, $F_{a c}$ and $F_{a c}$, in Fit 1 is compensated by the splitting of the $\Delta_{\mathrm{K}}$ distortion constant (the others are fixed) from the values in reference 12. A similar observation is made for the $\Delta_{\mathrm{JK}}$ and $\Delta_{\mathrm{K}}$ parameters for the $v_{13}$ band in Table 2 and is indicative of an untreated interaction.

Figure 5 shows a comparison of a region of the experimental spectrum of the Rbranch of TMS compared with that simulated using the constants for Fit 1 and the EA analyses presented in Table 1 . For the two tunneling components (blue: $0^{+} \rightarrow v_{13}{ }^{+}$, red: $0^{-}$ $\rightarrow v_{13^{-}}$), both model Hamiltonians clearly account for the strongest transitions which correspond to $\mathrm{K}_{\mathrm{a}}$ values up to 10 . The lower intensity transitions are not perfectly described by any of the methods presented and this may be a consequence of a small untreated perturbation as evidenced by the splitting of the $\Delta_{\mathrm{JK}}$ and $\Delta_{\mathrm{K}}$ parameters for the $v_{13}$ band in Table 2. Although interaction parameters for the $0^{+}$and $0^{-}$states were determined in the earlier rotational spectroscopic studies, ${ }^{10,12}$ the observed rovibrational spectra were not sensitive to the inclusion of these and their use led to only slight changes in the other spectroscopic parameters as compensation. This lends further support to our 
assertion that the parameters in Table 2 are simply 'effective' parameters and that the underlying physics of this vibrational band remains, as of yet, not fully understood. Many of the remaining, unassigned transitions in Figure 5 (and beyond) presumably correspond to those involving higher (perturbed) $\mathrm{K}_{\mathrm{a}}$ levels, however, there may be some contribution due to hotbands or weak $c$-type rotation-vibration-inversion transitions which are symmetry allowed. Although a few weak Q branches are observed (527.63, $528.16,530.7532 .15 \mathrm{~cm}^{-1}$ ), they do not appear strong enough to lead to a substantial number of $\mathrm{P}$ and $\mathrm{R}$ branch transitions with sufficient intensity.

We have also recorded the far infrared spectrum of the ring puckering mode of TMS at $139 \mathrm{~cm}^{-1}$ along with its hotbands using the CLS and expect that its analysis may lend further insight into the nature of the perturbation in the $v_{13}$ band. This is a very complex spectrum but as several excited vibrational states of the ring puckering mode have been characterized by rotational spectroscopy, ${ }^{10,11,12}$ it should be possible to better estimate the role of interaction terms for the ring inversion tunneling states involved. Further understanding of these problems will allow us to incorporate new terms into the EA code to allow automation of even more complex spectra in the future.

\section{Acknowledgements}

We extend our gratitude to B. Billinghurst for technical support at the Canadian

Light Source. This research was funded by the Natural Sciences and Engineering Research Council of Canada (NSERC) through the Discovery Grant (JvW: RGPIN/326993-2010) program. 
Table 1: Comparison of the spectroscopic constants for the $v_{16}$ band of azetidine from EA analysis and experiment.

\begin{tabular}{|c|c|c|c|c|}
\hline & \multicolumn{2}{|c|}{ Ground state } & \multicolumn{2}{|c|}{$v_{16}$} \\
\hline & EA & $\operatorname{Ref} 8^{a}$ & EA & $\operatorname{Ref} 8^{a}$ \\
\hline $\mathrm{E}$ & & & $207.7271513(130)$ & $207.7271511(84)$ \\
\hline $\mathrm{A} / \mathrm{cm}^{-1}$ & $0.3820398(20)$ & $0.3820399(11)$ & $0.3812483(22)$ & $0.3812484(11)$ \\
\hline B & $0.3782308(20)$ & $0.3782308(11)$ & $0.3776933(22)$ & $0.3776933(12)$ \\
\hline $\mathrm{C}$ & $0.22055329(24)$ & $0.22055329(16)$ & $0.21967014(24)$ & $0.21967014(16)$ \\
\hline$\Delta_{\mathrm{J}} / \mathrm{x} 10^{6} \mathrm{~cm}^{-1}$ & $0.16887(29)$ & $0.16884(79)$ & $0.14430(29)$ & $0.14428(90)$ \\
\hline$\Delta_{\mathrm{JK}}$ & $-0.13865(75)$ & $-0.1386(37)$ & $-0.05205(71)$ & $-0.0521(38)$ \\
\hline$\Delta_{\mathrm{K}}$ & $0.14894(60)$ & $0.1490(49)$ & $0.12799(55)$ & $0.1282(41)$ \\
\hline$\delta_{\mathrm{J}}$ & $0.0511(15)$ & $0.05109(39)$ & $0.0395(17)$ & $0.03947(45)$ \\
\hline$\delta_{\mathrm{K}}$ & $0.0631(11)$ & $0.0631(14)$ & $0.0672(11)$ & $0.0671(12)$ \\
\hline
\end{tabular}

${ }^{\mathrm{a}}$ Determined from single band analysis from 3278 rovibrational transitions described in Ref 8 with rms error $0.000130 \mathrm{~cm}^{-1}$. 
Table 2: Spectroscopic constants for the $v_{13}$ band of TMS from the various analyses described here.

\begin{tabular}{|c|c|c|c|c|c|c|}
\hline & \multicolumn{2}{|c|}{ EA } & \multicolumn{2}{|c|}{ SPFIT (Fit 1) } & \multicolumn{2}{|c|}{ SPFIT (Fit 2) } \\
\hline & $\mathrm{O}^{+}$ & $\mathbf{0}^{-}$ & $\mathrm{O}^{+}$ & $\mathbf{0}^{-}$ & $\mathrm{O}^{+}$ & $\mathbf{0}^{-}$ \\
\hline$E$ & 0 & $0.274589^{\mathrm{a}}$ & 0 & $0.274589^{\mathrm{a}}$ & 0 & $0.274589^{\mathrm{a}}$ \\
\hline $\mathrm{A} / \mathrm{cm}^{-1}$ & $0.3372453(12)$ & $0.3372438(12)$ & $0.33713984^{\mathrm{a}}$ & $0.33715131^{\mathrm{a}}$ & $0.33713984^{\mathrm{a}}$ & $0.33715131^{\mathrm{a}}$ \\
\hline $\mathrm{C}$ & $0.14813725(18)$ & $0.14815782(18)$ & $0.14813659(32)$ & $0.14816053(24)$ & $0.14822450(27)$ & $0.14824487(23)$ \\
\hline$\Delta_{\mathrm{J}} / \mathrm{x} 10^{6} \mathrm{~cm}^{-1}$ & $0.078703(39)$ & $\mathrm{b}$ & $0.078622(61)$ & $0.078886(39)$ & $0.079863(54)$ & $0.078959(39)$ \\
\hline$\Delta_{\mathrm{JK}}$ & $-0.07172(24)$ & $\mathrm{b}$ & $-0.07128^{a}$ & $-0.06681^{\mathrm{a}}$ & $-0.07128^{a}$ & $-0.06681^{a}$ \\
\hline$\delta_{\mathrm{J}}$ & $0.0202407^{\mathrm{a}}$ & $\mathrm{b}$ & $0.0202407^{\mathrm{a}}$ & $0.0204475^{\mathrm{a}}$ & $0.0202407^{\mathrm{a}}$ & $0.0204475^{\mathrm{a}}$ \\
\hline$\delta_{\mathrm{K}}$ & $0.064578^{\mathrm{a}}$ & $\mathrm{b}$ & $0.064578^{\mathrm{a}}$ & $0.0628768^{\mathrm{a}}$ & $0.064578^{\mathrm{a}}$ & $0.0628768^{\mathrm{a}}$ \\
\hline $\mathrm{F}_{\mathrm{ac}} / \mathrm{cm}^{-1}$ & & & & & \multicolumn{2}{|c|}{$0.00404783^{\mathrm{a}}$} \\
\hline $\mathrm{F}_{\mathrm{ac}} / \mathrm{x} 10^{6} \mathrm{~cm}^{-1}$ & & & & & \multicolumn{2}{|c|}{$-0.0198^{a}$} \\
\hline & $v_{13}{ }^{+}$ & $v_{13}^{-}$ & $v_{13}{ }^{+}$ & $v_{13^{-}}$ & $v_{13}{ }^{+}$ & $v_{13}-$ \\
\hline$E$ & $529.0213267(80)$ & $529.2619786(80)$ & $529.021271(17)$ & $529.262029(12)$ & $529.021172(17)$ & $529.261815(14)$ \\
\hline $\mathrm{A} / \mathrm{cm}^{-1}$ & $0.3374060(12)$ & $0.3374028(12)$ & $0.3372995(37)$ & $0.3373170(12)$ & $0.3373957(42)$ & $0.3373862(36)$ \\
\hline $\mathrm{C}$ & $0.14764237(18)$ & $0.147657809(18)$ & $0.14764176(32)$ & $0.14766037(25)$ & $0.14764289(27)$ & $0.14765829(23)$ \\
\hline$\Delta_{\mathrm{J}} / \mathrm{x} 10^{6} \mathrm{~cm}^{-1}$ & $0.078121(39)$ & $\mathrm{b}$ & $0.078003(61)$ & $0.078457(40)$ & $0.078311(54)$ & $0.077707(39)$ \\
\hline$\Delta_{\mathrm{IK}}$ & $-0.06598(40)$ & $\mathrm{b}$ & $-0.06251(39)$ & $-0.06938(16)$ & $-0.07981(43)$ & $-0.04217(21)$ \\
\hline$\Delta_{\mathrm{K}}$ & $0.2956(20)$ & $\mathrm{b}$ & $0.311(24)$ & $0.2070(95)$ & $0.316(17)$ & $0.1231(90)$ \\
\hline$\delta_{\mathrm{J}}$ & $0.0202407^{\mathrm{a}}$ & $0.0202407^{\mathrm{a}}$ & $0.0202407^{\mathrm{a}}$ & $0.0204475^{\mathrm{a}}$ & $0.0202407^{\mathrm{a}}$ & $0.0204475^{\mathrm{a}}$ \\
\hline$\delta_{\mathrm{K}}$ & $0.064578^{\mathrm{a}}$ & $0.064578^{\mathrm{a}}$ & $0.064578^{\mathrm{a}}$ & $0.0628768^{\mathrm{a}}$ & $0.064578^{\mathrm{a}}$ & $0.0628768^{\mathrm{a}}$ \\
\hline $\mathrm{rms} / \mathrm{cm}^{-1}$ & & & \multicolumn{2}{|c|}{0.000153} & \multicolumn{2}{|c|}{0.000124} \\
\hline no.lines & & & \multicolumn{2}{|c|}{2358} & \multicolumn{2}{|c|}{2358} \\
\hline
\end{tabular}

${ }^{\text {a }}$ Parameter fixed to result from analysis of rotational spectrum (reference 12).

${ }^{\mathrm{b}}$ Fixed to the corresponding values of the + state. 
Table 3: Comparison of spectroscopic parameters for the $0^{+}$and $0^{-}$states of TMS.

\begin{tabular}{|c|c|c|c|c|c|c|}
\hline & \multicolumn{2}{|c|}{ Reference 12} & \multicolumn{2}{|c|}{ EA } & \multicolumn{2}{|c|}{ SPFIT (Fit 1) } \\
\hline & $\mathbf{0}^{+}$ & $\mathbf{0}^{-}$ & $\mathbf{0}^{+}$ & $\mathbf{0}^{-}$ & $\mathbf{0}^{+}$ & $\mathbf{0}^{-}$ \\
\hline$E$ & 0 & $0.274589(1)$ & 0 & $0.274589^{a}$ & 0 & $0.274589^{a}$ \\
\hline $\mathrm{A} / \mathrm{cm}^{-1}$ & $0.33713984(3)$ & $0.33715131(3)$ & $0.3372453(12)$ & $0.3372438(12)$ & $0.33713984^{\mathrm{a}}$ & $0.33715131^{\mathrm{a}}$ \\
\hline $\mathrm{B}$ & $0.2224919(2)$ & $0.2224898(2)$ & $0.222604612(18)$ & $0.22237573(18)$ & $0.2226597(38)$ & $0.2223835(23)$ \\
\hline $\mathrm{C}$ & $0.14822312(2)$ & $0.14824556(2)$ & $0.14813725(18)$ & $0.14815782(18)$ & $0.14813659(32)$ & $0.14816053(24)$ \\
\hline$\Delta \mathrm{J} / \mathrm{x} 10^{6} \mathrm{~cm}^{-1} \mathrm{~b}$ & $0.079605(17)$ & $0.079155(33)$ & $0.078703(39)$ & $\mathrm{c}$ & $0.078622(61)$ & $0.078886(39)$ \\
\hline$\Delta_{\mathrm{JK}}$ & $-0.07128(33)$ & $-0.06681(37)$ & $-0.07172(24)$ & $\mathrm{c}$ & $-0.07128^{a}$ & $-0.06681^{a}$ \\
\hline$\Delta_{\mathrm{K}}$ & $0.224(1)$ & $0.218(7)$ & $0.2993(20)$ & $\mathrm{c}$ & $0.324(24)$ & $0.1717(94)$ \\
\hline$\delta_{\mathrm{J}}$ & $0.02024(1)$ & $0.02044(2)$ & $0.0202407^{a}$ & $\mathrm{c}$ & $0.0202407^{\mathrm{a}}$ & $0.0204475^{\mathrm{a}}$ \\
\hline$\delta_{\mathrm{K}}$ & $0.06458(3)$ & $0.06288(4)$ & $0.064578^{\mathrm{a}}$ & $\mathrm{c}$ & $0.064578^{\mathrm{a}}$ & $0.0628768^{\mathrm{a}}$ \\
\hline$\Phi_{\mathrm{JK}} / \mathrm{x} 10^{12} \mathrm{~cm}^{-1}$ & $-0.57(13)$ & $-0.2(1)$ & & & & \\
\hline$\phi_{\mathrm{JK}}$ & $0.67(20)$ & $0.47(23)$ & & & & \\
\hline $\mathrm{F}_{\mathrm{ad}} / \mathrm{cm}^{-1}$ & 0.00 & (17) & & & & \\
\hline $\mathrm{F}_{\mathrm{ac}} / \mathrm{x} 10^{6} \mathrm{~cm}^{-1}$ & & & & & & \\
\hline
\end{tabular}

${ }^{a}$ Parameter fixed to result from analysis of rotational spectrum (reference 12).

${ }^{\mathrm{b}}$ Centrifugal distortion parameters were calculated using B3LYP/6-311++g(2d,2p) for the ground state. For comparison, the values obtained $\left(\mathrm{x} 10^{6} \mathrm{~cm}^{-1}\right)$ were $: \Delta_{\mathrm{J}}=0.078384,: \Delta_{\mathrm{JK}}=-0.0560397, \Delta_{\mathrm{K}}=0.19757, \delta_{\mathrm{j}}=0.0201699, \quad \delta_{\mathrm{k}}=0.0732844$.

${ }^{\mathrm{c}}$ Fixed to the corresponding values of the + state. 
Figure 1: Equilibrium geometry of TMS in its principal inertial axis system.
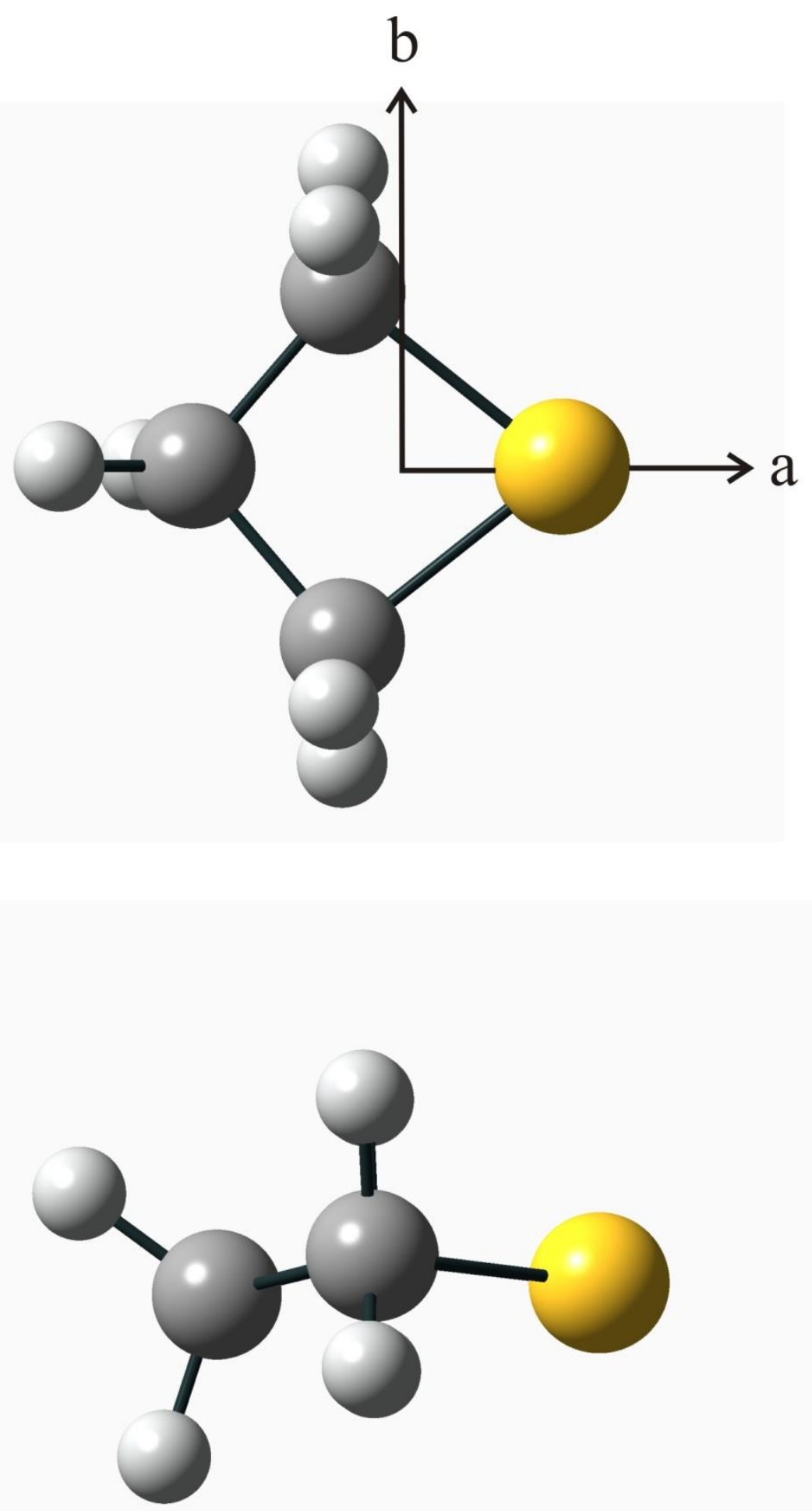
Figure 2: A closer look at a $0.25 \mathrm{~cm}^{-1}$ spectral region of the $\mathrm{R}$ branch of the $v_{16}$ band of azetidine. The simulation (blue lower trace) shows the expected $a$-type rotation-vibration transitions based on the EA analysis compared with the experimental spectrum (red upper trace). All predicted transitions are found in the experimental spectrum.
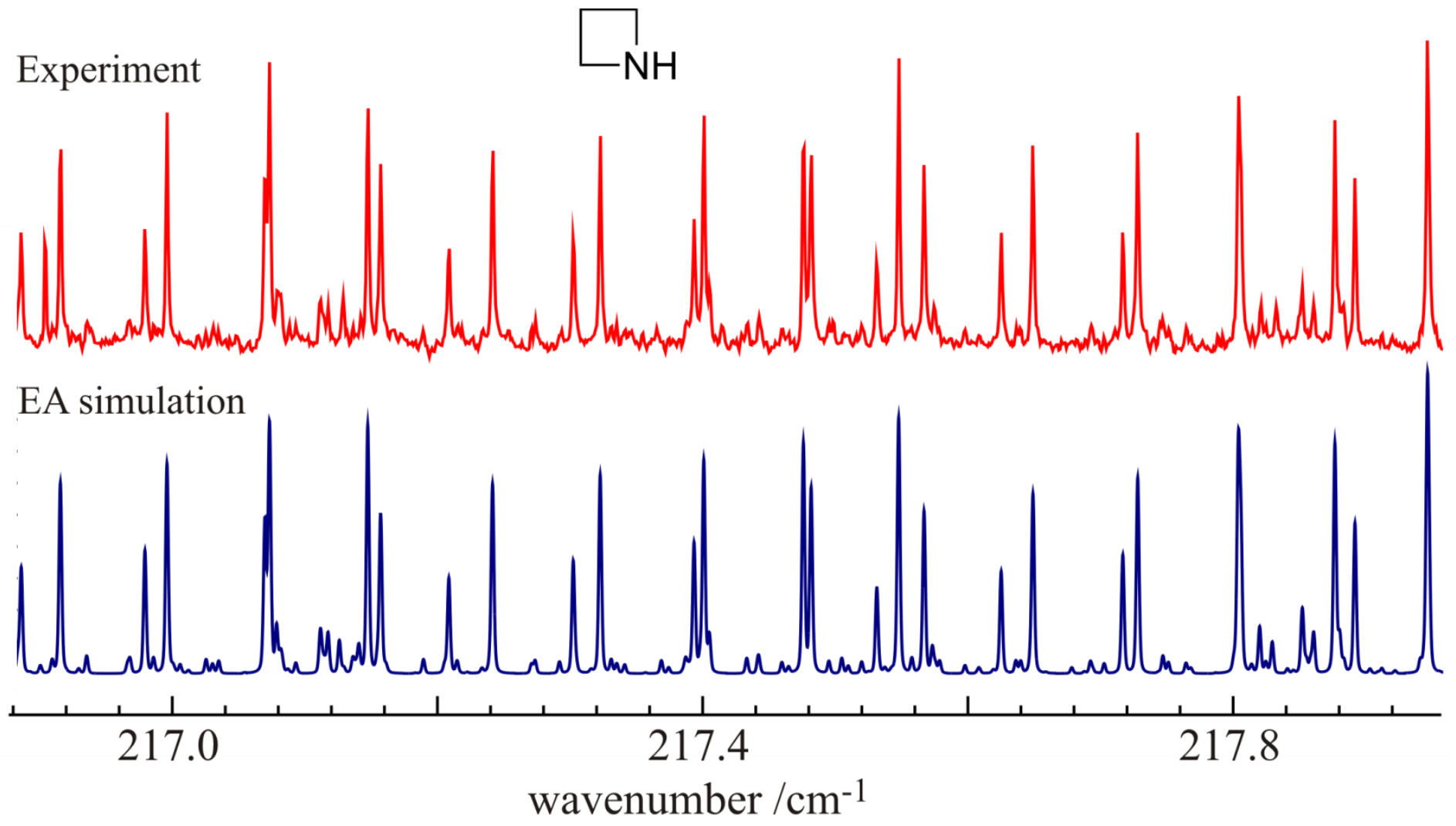
Figure 3: Overview spectrum showing band contour of the $v_{13}$ ring deformation mode of TMS at $\sim 529 \mathrm{~cm}^{-1}$.

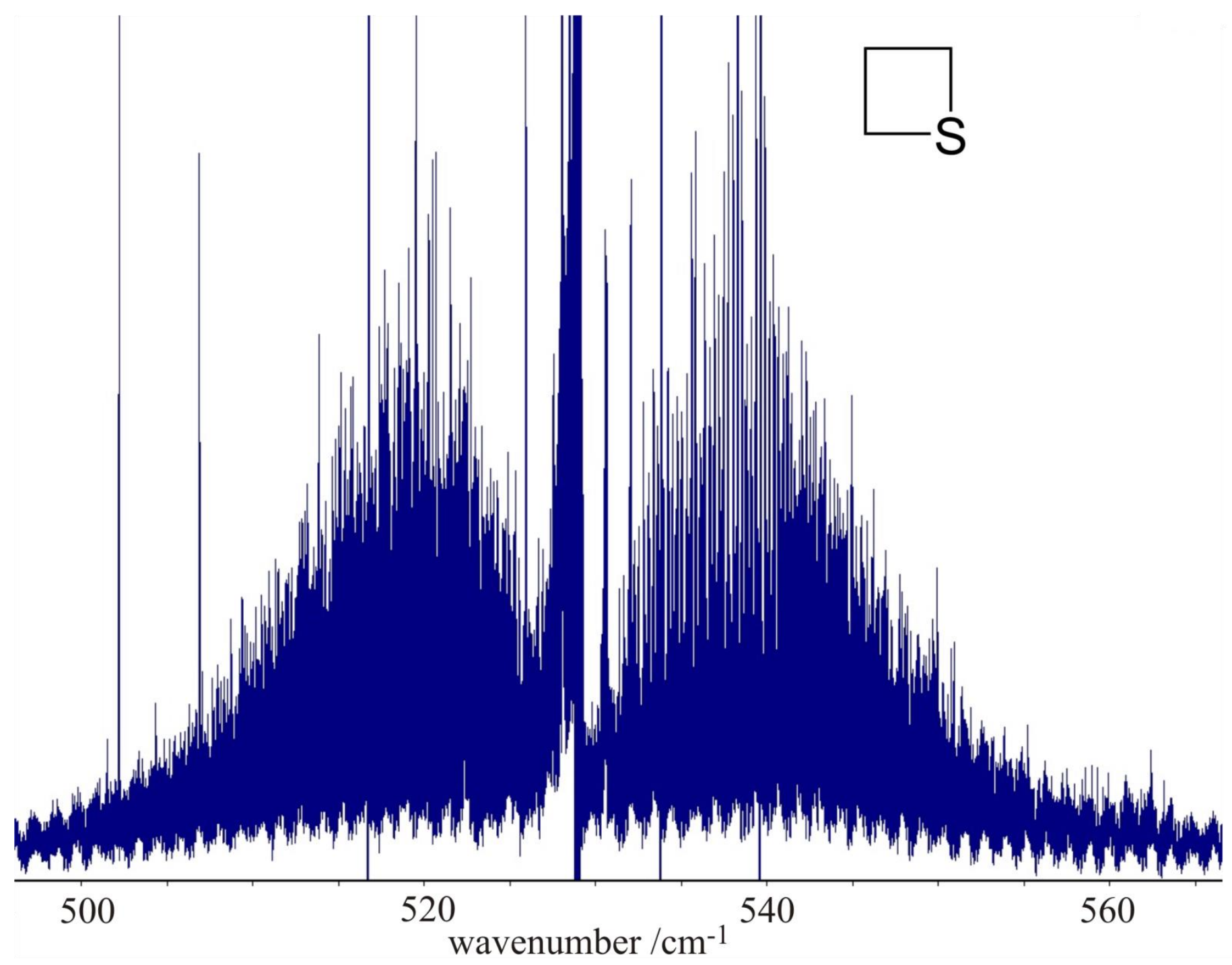


Figure 4: A portion of the Loomis-Wood plot of the $v_{13}$ band of TMS showing $a$-type progressions used to aid in its assignment. The coloured series identify transitions sharing common $\mathrm{K}_{\mathrm{a}}$ but having different $\mathrm{J}$ values with $0^{+} \rightarrow v_{13}{ }^{+}$and $0^{-} \rightarrow v_{13}{ }^{-}$, respectively. The coloured series correspond to $K_{a}=0$ (navy), $K_{a}=1$ (red), $K_{a}=2$ (yellow) and $K_{a}=3$ (green) progressions in each inversion component. Remaining series are not identified for simplicity.

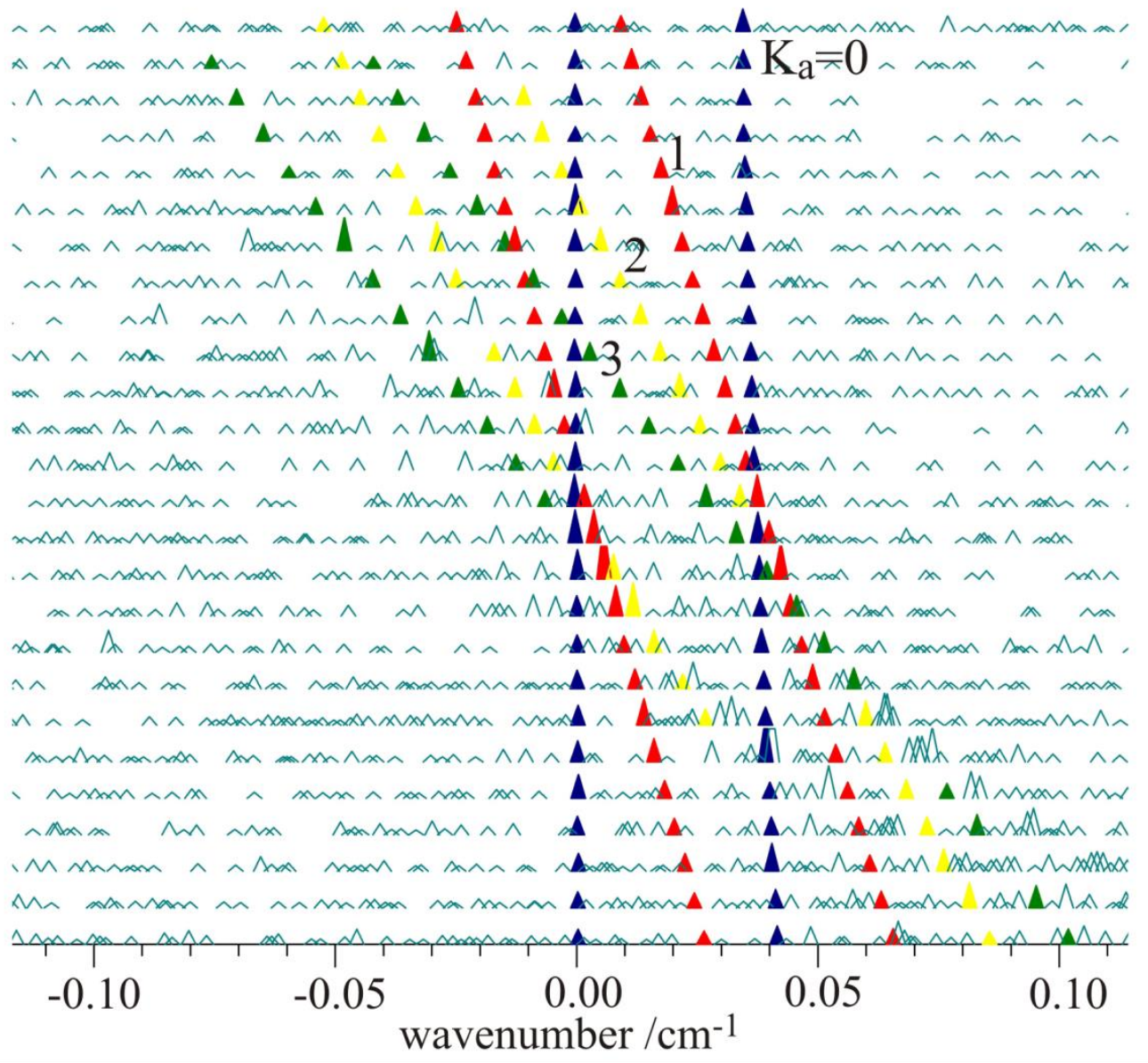


Figure 5: A closer look at a $0.4 \mathrm{~cm}^{-1}$ spectral region of the $\mathrm{R}$ branch of the $v_{13}$ band of TMS. The experimental spectrum (green top trace) is compared with simulated spectra using parameters derived in Fit 1 and using the EA approach. The strongest (low $\mathrm{K}_{\mathrm{a}}$ ) transitions are well-predicted by all three approaches. The blue trace corresponds to $a$-type transitions in the + inversion tunneling states and the red trace to those in the - states.
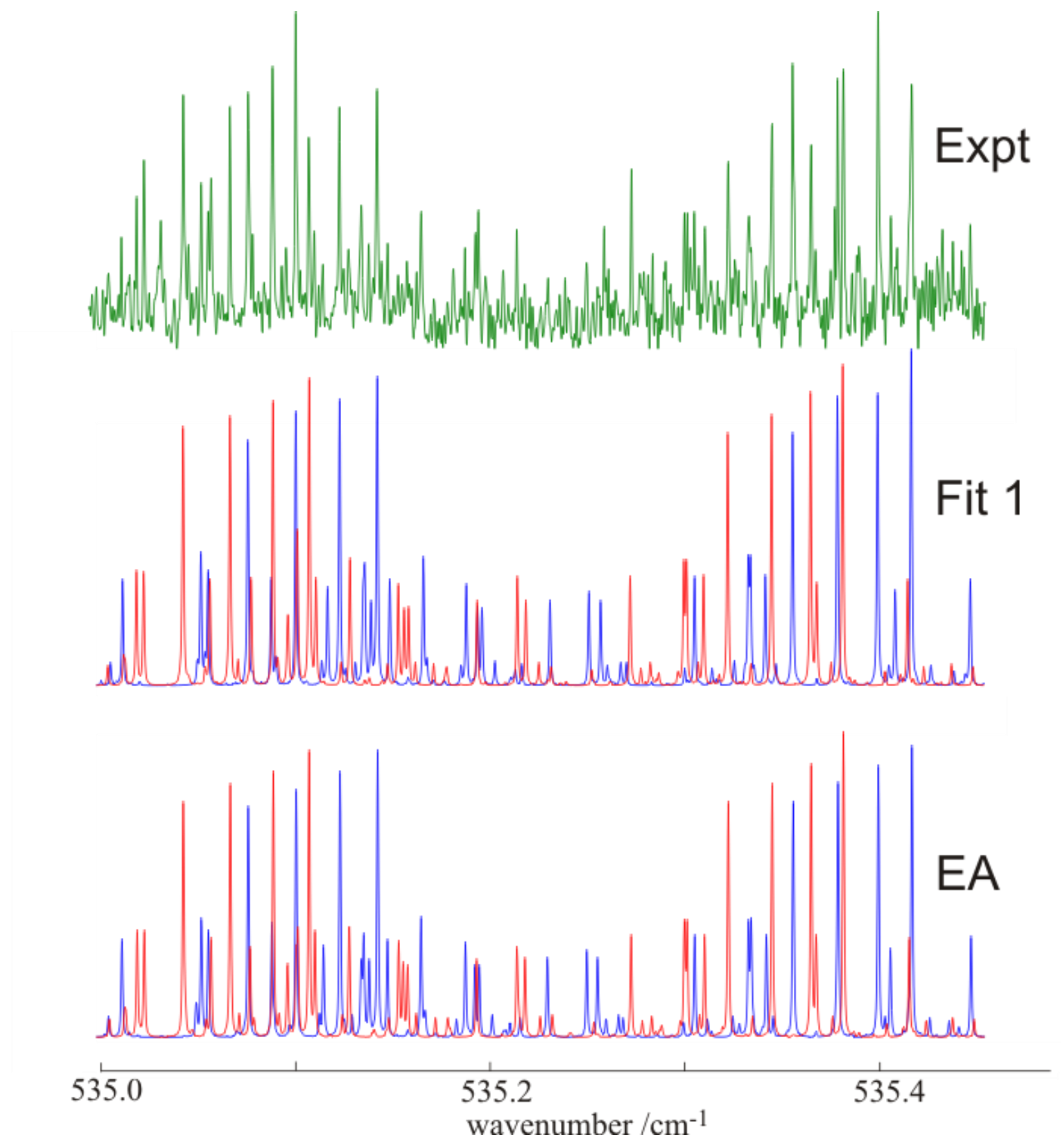


\section{References}

${ }^{1}$ W.L. Meerts, M. Schmitt, G.C. Groenenboom, Can. J. Chem. 82 (2004) 804-819.

${ }^{2}$ W. L. Meerts, M. Schmitt, Int. Rev. Phys. Chem. 25 (2006) 353-406.

${ }^{3}$ M. Schmitt, W.L. Meerts, In Handbook of High Resolution Spectroscopy, Volume II, page 1345. Edited by M. Quack and F. Merkt. John Wiley and sons, UK (2011). ISBN 978-0-47006653-9.

${ }^{4}$ M. Schmitt, L. Biemann, W. L. Meerts, K. Kleinermanns, J. Mol. Spectrosc. 257 (2009) 74-81.

${ }^{5}$ M. Motsch, M. Schenk, M. Zeppenfeld, M. Schmitt, W.L. Meerts, P.W.H. Pinkse, G. Rempe. J. Mol Spectrosc. 252 (2008) 25-30.

${ }^{6}$ W.L. Meerts, C.A. de Lange, A.C.J. Weber, E.E. Burnell. eMagRes 2013, Vol 2, (437 - 450).

${ }^{7}$ D.L.A.G. Grimminck, B. van Meerten, M.H.W. Verkuijlen, E.R.H. van Eck, W.L. Meerts, A.P.M. Kentgens. J. Magn. Resonance 228 (2013) 116 - 124.

${ }^{8}$ T. Zaporozan, Z. Chen, J. van Wijngaarden, J. Mol. Spectrosc. 264 (2010) 105-110.

${ }^{9}$ M.S. White, E.L. Beeson, J. Chem. Phys. 43 (1965) 1839-1841.

${ }^{10}$ D.O. Harris, H.W. Harrington, A.C. Luntz, W.D. Gwinn, J. Chem. Phys. 44 (1966) 3467-3480.

${ }^{11}$ R. Hinze, A. Guarnieri, J.L. Alonso, J.C. Lopez, J. Mol. Struct. 350 (1995) 195-204.

12 J.C. Lopez, A.G. Lessari, J.L. Alonso, R. Spiehl, A. Guarnieri, Mol. Phys. 82 (1994) 293-302.

${ }^{13}$ R.A. Shaw, C. Castro, N. Ibrahim, H. Wieser, J. Phys. Chem. 92 (1988) 6528-6536.

${ }^{14}$ D.M. Turnbull, M.G. Sowa, B.R. Henry, J. Phys. Chem. 100 (1996) 13433-13438.

15 T.R. Borgers, H.L. Strauss, J. Chem. Phys. 45 (1966) 947-955.

${ }^{16}$ J.R. Durig, R.C. Lord, J. Chem. Phys. 45 (1966) 61-66.

${ }^{17}$ M. Winnewisser, M. Kunzmann, M. Lock, B.P. Winnewisser, J. Mol. Struct. 561 (2001), 1-15.

18 A.R.W. McKellar, J. Mol. Spectrosc. J. Mol. Spectrosc. 262 (2010) 1-10 
${ }^{19}$ Z. Chen, J. van Wijngaarden, J. Mol. Spectrosc. 268 (2011) 107-111.

${ }^{20}$ Z. Chen, J. van Wijngaarden, J. Mol. Spectrosc. 279 (2012) 31-36.

${ }^{25}$ A. Ostermeier, A. Gawelczyk, and N. Hansen, Lecture Notes in Computer Science: Parallel Problem Solving from Nature (PPSN III) (Springer, 1994), p. 189.

${ }^{26}$ N. Hansen and A. Ostermeier, Evol. Comput. 9 (2001) 159.

${ }^{27}$ I. Kalkman, C. Vu, M. Schmitt, W.L. Meerts, Chem. Phys. Chem. 9 (2008) 1788.

${ }^{24}$ Z. Chen and J. van Wijngaarden, J. Mol. Spectrosc. 307 (2015) 10-17.

${ }^{25}$ C.F. Neese, Loomis-Wood Add-In (LWA) for Igor Pro, Version 2.0; 56th International Symposium on Molecular Spectroscopy. Columbus, OH, June 20-24 (2005) http://fermi.uchicago.edu/freeware/LoomisWood.shtml.

${ }^{26}$ IGOR Pro software, WaveMetrics Inc., Lake Oswego, OR USA, (2008).

${ }^{27}$ H. M. Pickett, J. Mol. Spectrosc. 148 (1991) 371-377; http://spec.jpl.nasa.gov/

${ }^{28}$ J. K. Watson, "Vibrational Spectra and Structure" (Ed: J. Durig) Vol 6. p 1, Elsevier, Amsterdam, 1977. 